H. Yokoi

Nagoya Math. J.

Vol. 134 (1994), 137-149

\title{
SOLVABILITY OF THE DIOPHANTINE EQUATION $x^{2}-D y^{2}= \pm 2$ AND NEW INVARIANTS FOR REAL QUADRATIC FIELDS
}

\author{
HIDEO YOKOI
}

In our recent papers $[3,4,5]$, we defined some new $D$-invariants for any square-free positive integer $D$ and considered their properties and interrelations among them. Especially, as an application of it, we discussed in [5] the characterization of real quadratic field $\mathbf{Q}(\sqrt{D})$ of so-called Richaud-Degert type in terms of these new $D$-invariants.

Main purpose of this paper is to investigate the Diophantine equation $x^{2}-$ $D y^{2}= \pm 2$ and to discuss characterization of the solvability in terms of these new $D$-invariants. Namely, we consider the equation $x^{2}-D y^{2}= \pm 2$ and first provide necessary conditions for the solvability by using an additive property and the multiplicative structure of $D$ (Proposition 2). Next, we provide necessary and sufficient conditions for the solvability in terms of an unit of the real quadratic field $\mathbf{Q}(\sqrt{D})$ (Theorems 1,2). Finally, we provide sufficient conditions for the solvability in terms of new $D$-invariants (Theorems 3,4 ). It is conjectured with a great expectation for these conditions to be also necessary conditions.

Throughout this paper, for any square-free positive integer $D$ we denote by $\varepsilon_{D}=\left(t_{D}+u_{D} \sqrt{D}\right) / 2(>1)$ the fundamental unit of the real quadratic field $\mathbf{Q}(\sqrt{D})$ and by $N$ the norm mapping from $\mathbf{Q}(\sqrt{D})$ to the rational number field $\mathbf{Q}$. Moreover, we denote $(/)$ the Legendre's symbol and by $[x]$ the greatest integer less than or equal to $x$.

On Pell's equation, we know already the following result by Perron (cf. [1], p. 106-109):

Proposition 1 (O. Perron). For any positive square-free integer $D \neq 2$, at most only one of the following three equations is solvable in integers:

Received April 19, 1993. 


$$
x^{2}-D y^{2}=-1, \quad x^{2}-D y^{2}=2, \quad x^{2}-D y^{2}=-2 .
$$

We may first provide the following necessary condition for solvability of the equation $x^{2}-D y^{2}= \pm 2$ :

Proposition 2. For any positive square-free integer $D$, if the Diophantine equation $x^{2}-D y^{2}= \pm 2$ has an integral solution, then

$$
D \equiv 2 \text { or } 3(\bmod 4) \text { and } N \varepsilon_{D}=1
$$

hold.

Moreover, if the equation $x^{2}-D y^{2}=2$ is solvable, then

$$
p \equiv \pm 1(\bmod 8)
$$

holds for any odd prime factor $p$ of $D$, and if the equation $x^{2}-D y^{2}=-2$ is solvable, then

$$
q \equiv 1 \text { or } 3(\bmod 8)
$$

holds for any odd prime factor $q$ of $D$.

Proof. When $x^{2}-D y^{2}= \pm 2$ has an integral solution $(x, y)=(a, b)$, if we assume $D \equiv 1(\bmod 4)$, then we get

$$
a^{2}-D b^{2} \equiv a^{2}-b^{2} \equiv 0 \text { or } \pm 1(\bmod 4) \text {, }
$$

which contradicts with $a^{2}-D b^{2}= \pm 2$.

Hence $D \equiv 2$ or $3(\bmod 4)$ holds.

On the other hand, if we assume $N \varepsilon_{D}=-1$, then the equation $x^{2}-D y^{2}=$ -1 is solvable, which contradicts with solvability of $x^{2}-D y^{2}= \pm 2$ by Proposition 1 . Hence $N \varepsilon_{D}=1$ holds.

Moreover, if the equation $x^{2}-D y^{2}=2$ is solvable, then for any odd prime factor $p$ of $D$, we get $(2 / p)=1$, and so $p \equiv \pm 1(\bmod 8)$ holds.

If the equation $x^{2}-D y^{2}=-2$ is solvable, then for any odd prime factor $q$ of $D$, we get $(-2 / q)=1$, and so $q \equiv 1$ or $(\bmod 8)$ holds.

Now we may provide the following necessary and sufficient conditions through an unit of the associated real quadratic field $\mathbf{Q}(\sqrt{D})$ with the equation $x^{2}-D y^{2}= \pm 2$ :

THEOREm 1. For any positive square-free integer $D$, it is necessary and sufficient 
for the equation $x^{2}-D y^{2}=2$ to be solvable that there exists an unit $\varepsilon=(t+$ $u \sqrt{D}) / 2>1$ of the real quadratic field $\mathbf{Q}(\sqrt{D})$ such that

$$
N \varepsilon=1 \text { and } t=D m+2
$$

for a positive integer $m$ satisfying $m \equiv 2(\bmod 8)$.

Proof. If the equation $x^{2}-D y^{2}=2$ has an integral positive solution

$$
(x, y)=\left(n_{1}, n_{2}\right) \text {, }
$$

i.e. $n_{1}^{2}-D n_{2}^{2}=2$ holds, then

$$
(t, u)=\left(2 n_{1}^{2}-2,2 n_{1} n_{2}\right)
$$

is an integral positive solution of the Diophantine equation $t^{2}-D u^{2}=4$, and hence $\varepsilon=(t+u \sqrt{D}) / 2>1$ is an unit of $\mathbf{Q}(\sqrt{D})$ and satisfies $N \varepsilon=1$.

Moreover, if we put $m=2 n_{2}{ }^{2}$, then

$$
t=2 n_{1}^{2}-2=D m+2
$$

holds, and from $n_{2} \equiv 1(\bmod 4)$ we get immediately

$$
m=2 n_{2}^{2} \equiv 2(\bmod 8) \text {. }
$$

Conversely, if there exists an unit $\varepsilon=(t+u \sqrt{D}) / 2>1$ of $\mathbf{Q}(\sqrt{D})$ such that $N \varepsilon=1$ and $t=D m+2$ for a positive integer $m$ satisfying $m \equiv 2(\bmod 8)$, then from $N \varepsilon=1$ we get

$$
D u^{2}=t^{2}-4=D(D m+4) m \text {, and so } u^{2}=(D m+4) m \text {. }
$$

On the other hand, $m \equiv 2(\bmod 8)$ implies $(D m+4, m)=2$. Hence, there exist two positive integers $n_{1}, n_{2}$ such that

$$
D m+4=2 n_{1}^{2}, m=2 n_{2}^{2}, \quad\left(\left(n_{1}, n_{2}\right)=1, u=2 n_{1} n_{2}\right),
$$

and hence $n_{1}{ }^{2}-D n_{2}{ }^{2}=2$ holds.

Therefore, the equation $x^{2}-D y^{2}=2$ has an integral positive solution

$$
(x, y)=\left(n_{1}, n_{2}\right) \text {. }
$$

For the equation $x^{2}-D y^{2}=-2$, we can prove the following analogous theorem:

THEOREM 2. For any positive square-free integer $D$, it is necessary and sufficient for the equation $x^{2}-D y^{2}=-2$ to be solvable that there exists an unit $\varepsilon=(t+$ 
$u \sqrt{D}) / 2>1$ of the real quadratic field $\mathbf{Q}(\sqrt{D})$ such that

$$
N \varepsilon=1 \text { and } t=D m-2
$$

for a positive integer $m$ satisfying $m \equiv 2(\bmod 8)$.

For any positive square-free integer $D$, we put

$$
\mathbf{A}_{D}=\left\{a: 0 \leqq a<D, a^{2} \equiv 4 N \varepsilon_{D}(\bmod D)\right\},
$$

and

$$
(A, B)_{\mathrm{D}}=\left\{(a, b): a \in \mathbf{A}_{D}, a^{2}-4 N \varepsilon_{D}=b D\right\} .
$$

Then, we obtained in [5] the following result:

There are uniquely determined non-negative integer $m_{D}$ and $\left(a_{D}, b_{D}\right)$ in $(A, B)_{D}$ such that

$$
\left\{\begin{array}{l}
t_{D}=D \cdot m_{D}+a_{D} \\
u_{D}{ }^{2}=D \cdot m_{D}{ }^{2}+2 a_{D} \cdot m_{D}+b_{D} .
\end{array}\right.
$$

Now, we may prove first the following:

Proposition 3. Under the assumption $D \neq 2,5$,

$$
a_{D}=2 \text { if and only if } b_{D}=0 \text {, }
$$

and

$$
a_{D}=D-2 \text { if and only if } b_{D}=D-4
$$

Proof. $a_{D}=2$ implies $b_{D} D={a_{D}}^{2}-4 N \varepsilon_{D}=4\left(1-N \varepsilon_{D}\right)$, and hence from $D \neq 2$, we get $N \varepsilon_{D}=1$ and $b_{D}=0$.

Conversely, $b_{D}=0$ implies

$$
a_{D}^{2}=b_{D} D+4 N \varepsilon_{D}=4 N \varepsilon_{D}
$$

and so we get

$$
N \varepsilon_{D}=1 \text { and } a_{D}=2 .
$$

Moreover, $a_{D}=D-2$ implies

$$
b_{D} D={a_{D}}^{2}-4 N \varepsilon_{D}=(D-2)^{2}-4 N \varepsilon_{D}=(D-4) D+4\left(1-N \varepsilon_{D}\right),
$$

and hence from $D \neq 2$, we get 


$$
N \varepsilon_{D}=1 \text { and } b_{D}=D-4 .
$$

Conversely, $b_{D}=D-4$ implies

$$
a_{D}{ }^{2}=b_{D} D+4 N \varepsilon_{D}=(D-4) D+4 N \varepsilon_{D}=(D-2)^{2}-4\left(1-N \varepsilon_{D}\right),
$$

and hence from $D \neq 5$, we get

$$
N \varepsilon_{D}=1 \text { and } a_{D}=D-2 .
$$

We can now provide the following sufficient conditions of the equation $x^{2}-$ $D y^{2}= \pm 2$ in terms of such invariants $a_{D}, b_{D}$ and $m_{D}$ :

THEOREM 3. If $\left(a_{D}, b_{D}\right)=(2,0)$ holds, then we have the following:

(1) $N \varepsilon_{D}=1$,

(2) $m_{D} \equiv 2(\bmod 8)$,

(3) $x^{2}-D y^{2}=2$ is solvable in integers.

Proof. We assume $\left(a_{D}, b_{D}\right)=(2,0)$, i.e.

$$
t_{D}=D m_{D}+2 \text { and } u_{D}{ }^{2}=D m_{D}{ }^{2}+4 m_{D} .
$$

Then, we can first get

$$
4 N \varepsilon_{D}=t_{D}^{2}-D u_{D}^{2}=4,
$$

and hence $N \varepsilon_{D}=1$.

Next, we assert $\left(D m_{D}+4, m_{D}\right)=2$.

If we assume $\left(D m_{D}+4, m_{D}\right)=1$, then it follows from $u_{D}^{2}=\left(D m_{D}+4\right) m_{D}$ that there exist two positive integers $n_{1}, n_{2}$ such that

$$
D m_{D}+4=n_{1}^{2}, m_{D}=n_{2}^{2} \text { with }\left(n_{1}, n_{2}\right)=1, u_{D}=n_{1} n_{2}
$$

and hence $n_{1}^{2}-D n_{2}^{2}=4$ holds.

However, since $n_{1}>1, u_{D}=n_{1} n_{2}$ is greater than $n_{2}$, which contradicts with minimum property of $u_{D}$.

If we assume $\left(D m_{D}+4, m_{D}\right)=4$, then similarly there exist two positive integers $n_{1}, n_{2}$ such that

$$
D m_{D}+4=4 n_{1}^{2}, m_{D}=4 n_{2}^{2} \text { with }\left(n_{1}, n_{2}\right)=1, u_{D}=4 n_{1} n_{2},
$$

and hence $n_{1}{ }^{2}-D n_{2}{ }^{2}=1$ holds. However, $u_{D}=4 n_{1} n_{2}$ is greater than $n_{2}$, which contradicts with minimum property of $u_{D}$.

Therefore, we get 


$$
\left(D m_{D}+4, m_{D}\right)=2 \text {, }
$$

and moreover it follows from $u_{D}{ }^{2}=\left(D m_{D}+4\right) m_{D}$ that there exist two positive integers $n_{1}, n_{2}$ such that

$$
D m_{D}+4=2 n_{1}{ }^{2}, m_{D}=2 n_{2}{ }^{2} \text { with } \quad\left(n_{1}, n_{2}\right)=1, u_{D}=2 n_{1} n_{2},
$$

and hence we get $n_{1}{ }^{2}-D n_{2}{ }^{2}=2$.

Furthermore, since $n_{2} \equiv 1(\bmod 2)$, we get finally

$$
m_{D}=2 n_{2}^{2} \equiv 2(\bmod 8) \text {. }
$$

Theorem 4. If $\left(a_{D}, b_{D}\right)=(D-2, D-4)$ holds, then we have the following:

$$
\begin{aligned}
& \text { (1) } N \varepsilon_{D}=1 \\
& \text { (2) } m_{D} \equiv 1(\bmod 8) \\
& \text { (3) } x^{2}-D y^{2}=-2 \text { is solvable in integers. }
\end{aligned}
$$

Proof. We assume $\left(a_{D}, b_{D}\right)=(D-2, D-4)$, i.e.

$$
t_{D}=D m_{D}+D-2 \text { and } u_{D}^{2}=D m_{D}{ }^{2}+2(D-2) m_{D}+D-4
$$

Then, we can first get

$$
4 N \varepsilon_{D}=t_{D}^{2}-D u_{D}^{2}=4,
$$

and hence we get $N \varepsilon_{D}=1$. Moreover, we get immediately

$$
u_{D}^{2}=\left(D m_{D}+D-4\right)\left(m_{D}+1\right) .
$$

Next, we assert $\left(D m_{D}+D-4, m_{D}+1\right)=2$.

If we assume $\left(D m_{D}+D-4, m_{D}+1\right)=1$, then it follows from $u_{D}{ }^{2}=\left(D m_{D}+D-4\right)\left(m_{D}+1\right)$ that there exist two positive integers $n_{1}, n_{2}$ such that

$$
D m_{D}+D-4=n_{1}^{2}, m_{D}+1=n_{2}^{2} \text { with }\left(n_{1}, n_{2}\right)=1, u_{D}=n_{1} n_{2},
$$

and hence $n_{1}{ }^{2}-D n_{2}{ }^{2}=-4$ holds, which contradicts with $N \varepsilon_{D}=1$.

If we assume $\left(D m_{D}+D-4, m_{D}+1\right)=4$, then similarly there exist two positive integers $n_{1}, n_{2}$ such that

$$
D m_{D}+D-4=4 n_{1}^{2}, m_{D}+1=4 n_{2}^{2} \text { with }\left(n_{1}, n_{2}\right)=1, u_{D}=4 n_{1} n_{2},
$$

and hence $n_{1}{ }^{2}-D n_{2}{ }^{2}=-1$ holds, which also contradicts with $N \varepsilon_{D}=1$.

Therefore, we get 


$$
\left(D m_{D}+D-4, m_{D}+1\right)=2 \text {. }
$$

Moreover, it follows from $u_{D}{ }^{2}=\left(D m_{D}+D-4\right)\left(m_{D}+1\right)$ that there exist two positive integers $n_{1}, n_{2}$ such that

$$
D m_{D}+D-4=2 n_{1}{ }^{2}, m_{D}+1=2 n_{2}{ }^{2} \text { with }\left(n_{1}, n_{2}\right)=1, u_{D}=2 n_{1} n_{2},
$$

and hence $n_{1}^{2}-D n_{2}{ }^{2}=-2$ holds.

Furthermore, since $n_{2} \equiv 1(\bmod 2)$, we get finally

$$
m_{D}=2 n_{2}^{2}-1 \equiv 1(\bmod 8) \text {. }
$$

Corollary 1. In the case $\left(a_{D}, b_{D}\right)=(2,0)$ (resp. $(D-2, D-4)$ ), the integral solution $(x, y)=\left(n_{1}, n_{2}\right)$ of the equation $x^{2}-D y^{2}=2$ (resp. $x^{2}-D y^{2}=$ -2) induced from the fundamental unit $\varepsilon_{D}$ of $\mathbf{Q}(\sqrt{D})$ in the proof of Theorem 3 (resp. 4) is the minimal positive solution.

Proof. In the case $\left(a_{D}, b_{D}\right)=(2,0)$, let $(x, y)=\left(n_{1}, n_{2}\right)$ be the integral solution induced from the fundamental unit $\varepsilon_{D}$ of $\mathbf{Q}(\sqrt{D})$, and $(x, y)=\left(m_{1}\right.$, $m_{2}$ ) be the minimal positive integral solution of the equation $x^{2}-D y^{2}=2$. Then,

$$
n_{1} \geqq m_{1}, n_{2} \geqq m_{2} \quad \text { and } \quad u_{D}=2 n_{1} n_{2}
$$

hold, and hence we get immediately

$$
u_{D} \geqq 2 m_{1} m_{2} .
$$

On the other hand, from the proof of Theorem 1

$$
(x, y)=\left(2 m_{1}^{2}-2,2 m_{1} m_{2}\right)
$$

is a positive integral solution of the equation $x^{2}-D y^{2}=4$, and hence we get $u_{D}$ $\leqq 2 m_{1} m_{2}$, by the minimum property of $u_{D}$. Therefore, we obtain $u_{D}=2 m_{1} m_{2}$, which implies $n_{1}=m_{1}, n_{2}=m_{2}$.

In the case $\left(a_{D}, b_{D}\right)=(D-2, D-4)$, we can also prove Corollary 1 in analogous way to the case $\left(a_{D}, b_{D}\right)=(2,0)$.

COROllary 2. If $D=q$ or $2 q$ for a prime number $q$ congruent to $3(\bmod 4)$, then $N \varepsilon_{D}=1$ holds.

Moreover, if $q \equiv-1(\bmod 8)$, then $a_{D}=2$ holds and $x^{2}-D y^{2}=2$ is solvable in integers.

If $q \equiv 3(\bmod 8)$, then $a_{D}=D-2$ holds and $x^{2}-D y^{2}=-2$ is solvable in integers. 
Proof. If we assume $N \varepsilon_{D}=-1$, then Pell's equation $x^{2}-D y^{2}=-4$ is solvable in integers, and so $q \equiv 1(\bmod 4)$ holds for any prime factor $q$ of $D$ which contradicts with $q \equiv 3(\bmod 4)$. Hence $N \varepsilon_{D}=1$ holds.

Next, since $t_{D}=D m_{D}+a_{D}, N \varepsilon_{D}=1$ implies

$$
D u^{2}=t_{D}{ }^{2}-4=m_{D}\left(D m_{D}+2 a_{D}\right) D+\left(a_{D}{ }^{2}-4\right),
$$

and hence

$$
\left(a_{D}-2\right)\left(a_{D}+2\right)=a_{D}{ }^{2}-4 \equiv 0(\bmod D) .
$$

Therefore, in the case $D=q$,

$$
a_{D} \equiv 2 \text { or }-2(\bmod D),
$$

and hence

$$
a_{D}=2 \text { or } D-2 .
$$

In the case $D=2 q, t_{D} \equiv 0(\bmod 2)$ implies $a_{D} \equiv 0(\bmod 2)$, and so

$$
a_{D}-2 \equiv a_{D}+2 \equiv 0 \text {, i.e. } a_{D} \equiv \pm 2(\bmod 2) .
$$

On the other hand, $a_{D} \equiv 2$ or $-2(\bmod q)$ holds, and so we get

$$
a_{D} \equiv 2 \text { or }-2(\bmod D)
$$

which implies directly

$$
a_{D}=2 \text { or } D-2 .
$$

Consequently, Corollary 2 is follows from Propositions 2,3 and Theorems 3.4. With regard to insolubility of $x^{2}-D y^{2}= \pm 2$, we obtain easily the following:

COROLLARY 3. If we assume

$$
D=p \quad \text { for a prime } p \text { congruent to } 1 \bmod 4 \text {, }
$$

or

$$
D=2 p \text { for a prime } p \text { congruent to } 5 \bmod 8
$$

then

$$
N \varepsilon_{D}=-1
$$

holds and 


$$
x^{2}-D y^{2}= \pm 2
$$

is insoluble.

Proof. If $D=p(p \equiv 1 \bmod 4)$, or $D=2 p(p \equiv 5 \bmod 8)$, then we get $N \varepsilon_{D}=-1$ (cf. for instance [2]).

Hence by Proposition $2 x^{2}-D y^{2}= \pm 2$ is insoluble.

$$
\left(a_{D}, b_{D}\right)=(2,0)
$$

\begin{tabular}{|c|c|c|c|c|c|c|}
\hline$D$ & type & $h_{D}$ & $r$ & $m_{D}$ & $n_{1}$ & $n_{2}$ \\
\hline 7 & $q$ & 1 & -2 & 2 & 3 & 1 \\
\hline 14 & $2 q$ & 1 & -2 & 2 & 4 & 1 \\
\hline 23 & $q$ & 1 & -2 & 2 & 5 & 1 \\
\hline 31 & $q$ & 1 & & 98 & 39 & 7 \\
\hline 34 & $2 p$ & 2 & -2 & 2 & 6 & 1 \\
\hline 46 & $2 q$ & 1 & & 1058 & 156 & 23 \\
\hline 47 & $q$ & 1 & -2 & 2 & 7 & 1 \\
\hline 62 & $2 q$ & 1 & -2 & 2 & 8 & 1 \\
\hline 71 & $q$ & 1 & & 98 & 59 & 7 \\
\hline 79 & $q$ & 3 & -2 & 2 & 9 & 1 \\
\hline 94 & $2 q$ & 1 & & 45602 & 1464 & 151 \\
\hline 103 & $q$ & 1 & & 4418 & 477 & 47 \\
\hline 119 & $p q$ & 2 & -2 & 2 & 11 & 1 \\
\hline 127 & $q$ & 1 & & 74498 & 2175 & 193 \\
\hline 142 & $2 q$ & 3 & -2 & 2 & 12 & 1 \\
\hline 151 & $q$ & 1 & & 22889378 & 41571 & 3383 \\
\hline 158 & $2 q$ & 1 & & 98 & 88 & 7 \\
\hline 167 & $q$ & 1 & -2 & 2 & 13 & 1 \\
\hline
\end{tabular}

$$
\begin{array}{ll}
t_{D}=D m_{D}+a_{D} & n_{1}=\sqrt{D \cdot m_{D} / 2+2} \\
u_{D}{ }^{2}=D m_{D}{ }^{2}+2 a_{D} m_{D}+b_{D} & n_{2}=\sqrt{m_{D} / 2} \\
a_{D}{ }^{2}-4=b_{D} D & t_{D}=D m_{D}+2 \\
& u_{D}=2 n_{1} \cdot n_{2}
\end{array}
$$




\begin{tabular}{|l|r|r|r|r|r|r|}
\hline$D$ & type & $h_{D}$ & $r$ & $m_{D}$ & $n_{1}$ & $n_{2}$ \\
\hline 191 & $q$ & 1 & & 94178 & 2999 & 217 \\
194 & $2 p$ & 2 & -2 & 2 & 14 & 1 \\
199 & $q$ & 1 & & 163479362 & 127539 & 9041 \\
206 & $2 q$ & 1 & & 578 & 244 & 17 \\
223 & $q$ & 3 & -2 & 2 & 15 & 1 \\
238 & $2 p q$ & 2 & & 98 & 108 & 7 \\
239 & $q$ & 1 & & 51842 & 2489 & 161 \\
254 & $2 q$ & 3 & -2 & 2 & 16 & 1 \\
263 & $q$ & 1 & & 1058 & 373 & 23 \\
287 & $p q$ & 2 & -2 & 2 & 17 & 1 \\
302 & $2 q$ & 1 & & 28322 & 2068 & 119 \\
311 & $q$ & 1 & & 108578 & 4109 & 233 \\
322 & $2 q 1$ & 2 & 18 & 1 \\
359 & $q$ & 4 & -2 & 2 & 19 & 1 \\
383 & $q$ & 3 & -2 & 98 & 137 & 7 \\
386 & $2 p$ & 2 & & 578 & 334 & 17 \\
391 & $p q$ & 2 & & 37538 & 2709 & 137 \\
398 & $2 q$ & 1 & -2 & 2 & 20 & 1 \\
431 & $q$ & 1 & & 703298 & 12311 & 593 \\
439 & $q$ & 5 & -2 & 2 & 21 & 1 \\
446 & $2 q$ & 1 & & 494018 & 10496 & 497 \\
479 & $q$ & 1 & & 12482 & 1729 & 79 \\
482 & $2 p$ & 2 & -2 & 2 & 1 \\
\hline
\end{tabular}

Prime $p$ is congruent to $1 \bmod 8 ; p \equiv 1(\bmod 8)$.

Prime $q$ is congruent to $-1 \bmod 8 ; q \equiv-1(\bmod 8)$.

$h_{D}=-n$ means that $N \varepsilon_{D}=-1$ and $h_{D}=n$.

$r$ represents the integer such that $D=k^{2}+r,-k<r \leqq k$ and $4 k \equiv 0(\bmod r)$ for real quadratic field $\mathbf{Q}(\sqrt{D})$ of $\mathbf{R}-\mathbf{D}$ type. 


$$
\left(a_{D}, b_{D}\right)=(D-2, D-4)
$$

$$
\begin{array}{ll}
t_{D}=D m_{D}+a_{D} & n_{1}=\sqrt{D\left(m_{D}+1\right) / 2-2} \\
u_{D}{ }^{2}=D m_{D}{ }^{2}+2 a_{D} m_{D}+b_{D} & n_{2}=\sqrt{\left(m_{D}+1\right) / 2} \\
a_{D}{ }^{2}-4=b_{D} D & t_{D}=D\left(m_{D}+1\right)-2 \\
& u_{D}=2 n_{1} \cdot n_{2}
\end{array}
$$

\begin{tabular}{|c|c|c|c|c|c|c|}
\hline$D$ & type & $h_{D}$ & $r$ & $m_{D}$ & $n_{1}$ & $n_{2}$ \\
\hline 2 & 2 & -1 & -2 & 1 & & 1 \\
\hline 3 & $q$ & 1 & -2 & 1 & 1 & 1 \\
\hline 6 & $2 q$ & 1 & 2 & 1 & 2 & 1 \\
\hline 11 & $q$ & 1 & 2 & 1 & 3 & 1 \\
\hline 19 & $q$ & 1 & & 17 & 13 & 3 \\
\hline 22 & $2 q$ & 1 & & 17 & 14 & 3 \\
\hline 38 & $2 q$ & 1 & 2 & 1 & 6 & 1 \\
\hline 43 & $q$ & 1 & & 161 & 59 & 9 \\
\hline 51 & $p q$ & 2 & 2 & 1 & 7 & 1 \\
\hline 59 & $q$ & 1 & & 17 & 23 & 3 \\
\hline 66 & $2 q_{1} q_{2}$ & 2 & 2 & 1 & 8 & 1 \\
\hline 67 & $q$ & 1 & & 1457 & 221 & 27 \\
\hline 83 & $2 q$ & 1 & 2 & 1 & 9 & 1 \\
\hline 86 & $2 q$ & 1 & & 241 & 102 & 11 \\
\hline 102 & $2 p q$ & 2 & 2 & 1 & 10 & 1 \\
\hline 107 & $q$ & 1 & & 17 & 31 & 3 \\
\hline 114 & $2 q_{1} q_{2}$ & 2 & & 17 & 32 & 3 \\
\hline 118 & $2 q$ & 1 & & 5201 & 554 & 51 \\
\hline 123 & $p q$ & 1 & & 1 & 11 & 1 \\
\hline 131 & $q$ & 1 & & 161 & 103 & 9 \\
\hline 134 & $2 q$ & 1 & & 2177 & 382 & 33 \\
\hline 139 & $q$ & 1 & & 1116017 & 8807 & 747 \\
\hline 146 & $2 p$ & 2 & 2 & 1 & 12 & 1 \\
\hline 163 & $q$ & 1 & & 786257 & 8005 & 627 \\
\hline 178 & $2 p$ & 2 & & 17 & 40 & 3 \\
\hline 179 & $q$ & 1 & & 46817 & 2047 & 153 \\
\hline 187 & $p q$ & 2 & & 17 & 41 & 3 \\
\hline 211 & $q$ & 1 & & & & \\
\hline
\end{tabular}

$$
m_{D}=\left[t_{D} / D\right]=2 n_{2}^{2}-1 \equiv 1(\bmod 8) \quad n_{1}{ }^{2}-D n_{2}{ }^{2}=-2
$$




\begin{tabular}{|c|c|c|c|c|c|c|}
\hline$D$ & type & $h_{D}$ & $r$ & $m_{D}$ & $n_{1}$ & $n_{2}$ \\
\hline 214 & $2 q$ & 1 & & & & \\
\hline 227 & $q$ & 1 & 2 & 1 & 15 & 1 \\
\hline 246 & $2 p q$ & 2 & & 721 & 298 & 19 \\
\hline 251 & $q$ & 1 & & 29281 & 1917 & 121 \\
\hline 258 & $2 p q$ & 2 & & 1 & 16 & 1 \\
\hline 262 & $2 q$ & 1 & & 801377 & 10246 & 633 \\
\hline 267 & $p q$ & 2 & & 17 & 49 & 3 \\
\hline 278 & $2 q$ & 1 & & 17 & 50 & 3 \\
\hline 283 & $q$ & 1 & & 977201 & 11759 & 699 \\
\hline 291 & $p q$ & 4 & 2 & 1 & 17 & 1 \\
\hline 307 & $q$ & 1 & & 576737 & 9409 & 537 \\
\hline 326 & $2 q$ & 3 & & 1 & 18 & 1 \\
\hline 339 & $p q$ & 2 & & 577 & 313 & 17 \\
\hline 347 & $q$ & 1 & & 3697 & 801 & 43 \\
\hline 354 & $2 q_{1} q_{2}$ & 2 & & 1457 & 508 & 27 \\
\hline 358 & $2 q$ & 1 & & & & \\
\hline 374 & $2 p q$ & 2 & & 17 & 58 & 3 \\
\hline 402 & $2 q_{1} q_{2}$ & 2 & & 1 & 20 & 1 \\
\hline 411 & $p q$ & 2 & & 241 & 223 & 11 \\
\hline 418 & $2 q_{1} q_{2}$ & 2 & & 161 & 184 & 9 \\
\hline 419 & $q$ & 1 & & 1289617 & 16437 & 803 \\
\hline 422 & $2 q$ & 1 & & 33281 & 2650 & 129 \\
\hline 443 & $q$ & 3 & 2 & 1 & 21 & 1 \\
\hline 451 & $p q$ & 2 & & 206081 & 6817 & 321 \\
\hline 454 & $2 q$ & 1 & & & & \\
\hline 467 & $q$ & 1 & & 6961 & 1275 & 59 \\
\hline 498 & $2 q_{1} q_{2}$ & 2 & & 721 & 424 & 19 \\
\hline 499 & $q$ & 5 & & 17 & 67 & 3 \\
\hline
\end{tabular}

Prime $p$ is congruent to $1 \bmod 8 ; p \equiv 1(\bmod 8)$

Prime $q$ is congruent to $3 \bmod 8 ; q \equiv 3(\bmod 8)$.

\section{REFERENCES}

[1] O. Perron, Die Lehre von den Kettenbruchen, Chelsea Publ. Comp., 1929.

[2] T. Takagi, Syoto-sesuron-kogi (Japanese), Kyoritu Publ. Comp., 1953.

[3] H. Yokoi, Some relations among new invariants of prime number $p$ congruent to 1 mod 4, Advances in Pure Math.,13 (1988), 493-501. 
[4] The fundamental unit and bounds for class numbers of real quadratic fields, Nagoya Math. J., 124 (1991), 181-197.

[5] - New invariants and class number problem in quadratic fields, Nagoya Math. J., 132 (1993), 175-197.

Graduate School of Human Informatics

Nagoya University

Chikusa-ku, Nagoya 464-01

Japan 